\title{
A ABORDAGEM DIALÓGICA PARA A FORMAÇÃO ÉTICA DO ENFERMEIRO NO PROCESSO DE DOAÇÃO DE ÓRGÃOS
}

\author{
Elton Carlos de Almeida ${ }^{1}$ \\ Sonia Maria Villela Bueno ${ }^{2}$ \\ Vanessa Denardi Antoniassi Baldissera ${ }^{3}$
}

ALMEIDA, E. C. de; BUENO, S. M. V.; BALDISSERA, V. D. A. A abordagem dialógica para a formação ética do enfermeiro no processo de doação de órgãos. Arq. Ciênc. Saúde Unipar, Umuarama, v. 18 n. 1, p. 19-22, jan./abr. 2014

\begin{abstract}
RESUMO: Considerando necessário analisar a formação, o preparo e o enfrentamento dos discentes de enfermagem nas questões éticas que envolvem o processo de doação de órgãos, utilizou-se a pesquisa-ação. Foi aplicado um questionário para 18 alunos do curso de graduação em Enfermagem de uma instituição pública do Estado do Paraná-Brasil, seguindo as normas brasileiras para experimentação humana. Diante dos resultados, codificamos os conteúdos programáticos em reflexões éticas a respeito da captação/doação de órgãos, executando e avaliando a ação educativa nessa temática. A doação de órgãos foi descrita como uma decisão nobre, por relacionar-se com a benevolência e o altruísmo, e corroboram a incipiência da temática durante sua formação. Evidenciou-se saberes acríticos e incipientes, sobretudo nas questões éticas. Por meio da ação educativa foi realizado uma abordagem dialógica, pautadas no referencial de Paulo Freire, levantando questões que evidenciassem conceitos de vida/morte e os princípios éticos envolvidos, permitindo a descodificação e o desvelamento crítico pelos alunos. Os participantes relataram que a ação educativa permitiu alavancar seus saberes no entorno da temática e que reflexões dessa natureza são imprescindíveis para a formação ética do enfermeiro, indissociáveis do saber técnico-científico. Destarte, o tema doação de órgãos permitiu abordar de forma mais pontual as questões bioéticas que permeiam esse processo, além de colaborar para a formação ética, crítica e contextualizada do enfermeiro.
\end{abstract}

PALAVRAS-CHAVE: Enfermagem; Ética; Educação.

\section{THE DIALOGICAL APPROACH FOR NURSING ETHICS TRAINING IN THE PROCESS OF ORGAN DONATION}

ABSTRACT: Considering that it is necessary to analyze the training, preparation and coping techniques from nursing students on ethical issues involving the process of organ donation, an action research was used in this study. A questionnaire was applied to 18 nursing undergraduate students in a public institution in the state of Paraná, Brazil, following the Brazilian rules for human experimentation. Given the results, the syllabus was coded into ethical reflections on the organ harvesting/donation. Educational activities were executed and evaluated on this theme. Organ donation was described as a noble decision, related to benevolence and altruism, which corroborates to the paucity of this subject during undergraduate education. Uncritical and incipient knowledge was evidenced, particularly on ethical issues. Through the educational activity, a dialogical approach was performed, based on Paulo Freire, where it was possible to raise questions showing concepts of life/death and the ethical principles involved, allowing the process of decoding and critical unveiling of the students. The participants reported that the educational activity allowed them to leverage their knowledge on the theme and that reflections of this nature are essential for ethical nursing education, which should not be separable from the technical and scientific knowledge. Thus, the topic of organ donation allowed us to punctually analyze the bioethical issues permeating such process, and contribute to the ethical, critical and contextualized nursing education.

KEYWORDS: Nursing; Ethics; Education.

\section{Introdução}

Os progressos científicos, tecnológicos e organizacionais colaboraram de forma considerável para o aumento do número de transplante de órgãos e tecidos, melhorando a qualidade de vida de muitas pessoas que esperavam por um órgão. Porém, o número de doadores insuficiente para reduzir a lista de espera continua sendo um desafio e constitui-se como o maior obstáculo para realização dos transplantes (SANTOS; MASSAROLLO; MORAES, 2012).

$\mathrm{Na}$ perspectiva de aumentar o índice de doação, Vieira (2006) enfatizou a necessidade de ampliações nas divulgações da importância da doação de órgãos, especialmente quanto aos aspectos técnicos e éticos que a envolvem, uma vez que grande parte da população brasileira desconfia da conduta bioética dos profissionais das equipes intra-hospitalares. A recusa dos familiares é motivo que afeta a doação de órgãos, ressaltando que a decisão pode estar ligada ao desempenho profissional na identificação e notificação de possíveis doadores, bem como no acolhimento à família, postulando questões éticas no entorno dessa prática. Dessa maneira, a bioética pode ser um instrumento para implementação de uma prática segura no processo de doação/transplante de órgãos e tecidos (ROZA; SCHIRMER, 2007).

Assim, vale destacar a importância do preparo profissional no que diz respeito à abordagem familiar, esclarecendo as dúvidas que podem surgir sobre morte encefálica e a solicitação para doação de órgãos, configurando-se o mo-

DOI: https://doi.org/10.25110/arqsaude.v18i1.2014.5153

${ }^{1}$ Enfermeiro. Mestre e Doutorando em Ciências pelo Departamento de Enfermagem Psiquiátrica e Ciências Humanas - DPCH da Escola de Enfermagem de Ribeirão Preto - EERP/USP. Membro Efetivo do Grupo de Pesquisa CAESOS. Av. dos Bandeirantes, 3900, Campus Universitário, Bairro Monte Alegre, 14040-902. Fone: (16) 98111-7244. Ribeirão Preto, SP, Brasil. ecarlos23@gmail.com

${ }^{2}$ Pedagoga. Doutora em Educação. Professora Associada Nível III. Livre Docente do DPCH da EERP/USP. Av. dos Bandeirantes, 3900, Campus Universitário, Bairro Monte Alegre, 14040-902. Fone: (16) 3602 3524. E-mail: smvbueno@eerp.usp.br. CAESOS - Centro Avançado de Educação para a Saúde e Orientação Sexual - Educação Preventiva em Sexualidade, DST, AIDS, Drogas e Violência. GEPES - Grupo de Estudos e Pesquisas em Educação e Saúde - UEM.

${ }^{3}$ Enfermeira, Doutora em Ciências, Professora do Departamento de Enfermagem da Universidade Estadual de Maringá (UEM). Av. Colombo - Jardim Universitário, 87050-390, Maringá, Pr. Fone: (44) 30114815, e-mail: vanessadenardi@hotmail.com. CAESOS - Centro Avançado de Educação para a Saúde e Orientação Sexual - Educação Preventiva em Sexualidade, DST, AIDS, Drogas e Violência. GEPES - Grupo de Estudos e Pesquisas em Educação e Saúde - UEM 
mento importante para que se possa dar continuidade ao processo de captação de órgãos (ALMEIDA, 2011; SANTOS; MASSAROLLO; MORAES, 2012). O sucesso da assistência à família e ao paciente se faz com os profissionais que, de certa forma, encontrem-se preparados na questão de conhecimentos científicos e habilidades para o enfrentamento de emoções (CINQUE; BIANCHI, 2010).

A doação de órgãos e transplantes de órgãos e tecido é tema polêmico que gera inúmeras discussões, sendo que a falta de informações sobre o assunto e a ausência de programas de sensibilização contribui para o desconhecimento, gerando mitos e preconceitos (MORAIS; MORAIS, 2012), sobretudo entre profissionais de saúde. Também o desenvolvimento tecnológico que possibilitou avanços no processo de doação de órgãos e transplante tem desencadeado diversos dilemas éticos que os permeiam. Em decorrência, as discussões bioéticas tornam-se constantes e pertinentes (LIMA, 2007). Pressupõe-se que haja prudência entre o conhecimento técnico-científico associado aos valores humanos (DINIZ; GUILHEM, 2002), especialmente nas questões específicas do processo de doação de órgãos.

Diante do panorama apresentado, interessava-nos saber qual o preparo dos futuros enfermeiros de uma instituição pública do Estado do Paraná-Brasil, quanto às questões éticas que envolvem o processo de doação de órgãos e tecidos.

Desta forma, o presente estudo teve por objetivo analisar a formação, o preparo e o enfrentamento dos discentes de enfermagem nas questões éticas que envolvem o processo de doação de órgãos, permitindo a elaboração, implantação e avaliação de ação educativa nessa temática.

\section{Material e Método}

Tratou-se de uma investigação qualitativa, humanista, mediatizada pelo método da pesquisa-ação, pelo qual realizamos o levantamento temático, elaboramos, desenvolvemos e avaliamos a ação educativa na perspectiva das questões éticas que envolvem o processo de doação de órgãos.

O referencial teórico-metodológico adotado baseia-se nos pressupostos de Freire (2011) que delimita o método em três partes: levantamento de temas geradores, codificação/descodificação e desvelamento crítico. O levantamento de dados sócio-demográficos e da percepção temática ocorreu por meio da aplicação de questionários contendo questões abertas que permitiram elencar os temas geradores usando categorização temática; na sequência, a ação educativa se deu a partir do tratamento didático dos temas geradores, transformando-os em conteúdos programáticos que foram codificados/descodificados e desvelados criticamente pelos participantes por meio de círculos de cultura. Os diários de campo colaboraram para nossa apreensão sobre o processo formativo e o uso de relatórios, pelos participantes, permitiu nossa avaliação somativa.

A população deste estudo se constituiu de 18 discentes de enfermagem de uma Universidade Estadual do Paraná-Brasil que foram selecionados após apreciação do Comitê de Ética em Pesquisa envolvendo Seres Humanos (Parecer n²17.254 COPEP-UEM), autorização expressa da Direção do Curso a que estão vinculados por meio da assinatura do Termo de Permissão de Utilização dos Dados e mediante assinatura do Termo de Consentimento Livre e Esclarecido pelos participantes, cumprindo com os preceitos da experimentação humana definidos pela Resolução 466/12 do Conselho Nacional de Saúde do Brasil.

Cumpre destacar que esse trabalho faz parte de investigação conduzida pelo 'Grupo de Estudos e Pesquisas em Educação e Saúde' cadastrado no Diretório de Pesquisas do CNPQ/Brasil, está vinculado à Pesquisa Institucional da Universidade Estadual de Maringá intitulada 'Pesquisa-ação nas demandas educativas no cenário da enfermagem' e constitui-se de resultados que integrarão uma tese de doutorado em elaboração.

\section{Resultados}

Optamos por apresentar os resultados seguindo as três partes descritas no referencial teórico-metodológico: levantamento de temas geradores, codificação/descodificação e desvelamento crítico.

\section{Levantamento de tema gerador}

Na percepção dos participantes, a doação de órgãos é uma decisão nobre, tanto para o doador quanto para o profissional que atua nesta área, por relacionar-se com a benevolência e o altruísmo. Tal percepção foi analisada revelando o tema gerador para a ação educativa: as questões que envolvem a vida, como seus princípios e valores, que poderiam ser discutidas embasadas na bioética inerente ao processo de doação de órgãos.

\section{Codificação/Descodificação}

Diante da definição do tema gerador codificamos os conteúdos programáticos em situações que possibilitariam sua descodificação por meio de reflexões éticas a respeito da captação/doação de órgãos. Assim, executamos e avaliamos a ação educativa nessa temática. A descodificação ocorreu durante a ação educativa organizada em círculos de cultura, permeada por aula dialogada com situações que suscitaram discussões acerca da temática.

É preciso apontar que na Codificação e Descodificação ocorrereu a análise e representação da investigação temática, apresentando as situações-problemas reais, que levaram à reflexão da própria realidade, para, na sequência, descodificá-las e reconhecê-las em um processo crítico.Optamos por começar esclarecendo aos participantes sobre a ética, para então, abordarmos a bioética da vida pontuando seus princípios e a importância de compreender cada um desses durante o processo de doação/captação de órgãos. Apresentamos a ética como mecanismo de regulação das relações sociais, que tem por objetivo garantir a união social e harmonizar interesses individuais e coletivos (OSELKA, 2008).

No que tange à bioética, iniciamos com sua trajetória e origem da palavra que vem do grego, sendo, bios: vida; ethos: ética. Afirmamos ser a bioética a ciência que faz o estudo sistemático da conduta humano, nas áreas das ciências da vida e dos cuidados à saúde, na medida que essa conduta é encaminhada e baseada nos valores e princípios morais da sociedade (REICH, 1995).

Embasando-se na bioética levantamos alguns assun- 
tos polêmicos para inserir os alunos em situações que pudessem compreender e discutir tais temáticas com embasamento no principialismo. A partir de então, levamos à discussão para questões que envolvem o processo de doação/captação de órgãos, articulando-as com os princípios da bioética.

As discussões acerca das células-troncos foram utilizadas para suscitar reflexões a respeito do início da vida. Tal conceito pode influenciar, de certa forma, o processo de doação de órgãos; considerando que a vida começa quando o sistema circulatório inicia sua função pode questionar-se a morte encefálica: se há presença dos batimentos cardíacos e consequentemente funções de irrigação corpórea, ainda há vida em um paciente em morte encefálica? Essa foi uma questão levantada no grupo para que pudéssemos refletir e pensar de forma crítica e reflexiva, pois de certa maneira, tais questões fazem parte do cotidiano do profissional de saúde, principalmente daqueles que atuam frente à doação de órgãos.

Da mesma forma, instigamos a discussão ao grupo tomando por verdade o início da vida após o funcionamento do sistema nervoso central, justificando positivamente a doação por um potencial doador em morte encefálica. Essa compreensão de que não há vida em pacientes nessa condição é fato e tal situação foi levantada para darmos continuidade ao círculo de cultura, permitindo desvelamento crítico da situação em pauta.

Outras questões discutidas foram a eutanásia, a distanásia e a ortotanásia, pois, dependendo do conhecimento dos familiares a atitude do profissional pode ser interpretado de forma errônea. As situações discutidas levaram o grupo a refletir sobre as questões que podem evidenciar os princípios da bioética que são a autonomia, a beneficência e a justiça. Esclarecemos sobre cada um desses e, em relação à autonomia, levamos à reflexão de que o indivíduo só é capaz de agir com autonomia se houver consciência crítica do assunto. Para isso, receber as informações necessárias é imprescindível para que possa agir, realmente, de forma autônoma, transformando sua visão ingênua pela visão crítica (FREIRE, 2011).

Fernandez (2000) e Lima (2012) referindo-se à autonomia insistem que todas as práticas ou atitudes que derivem ou expressem uma concepção instrumental do ser humano, em transplantes devem ser rejeitadas. A autonomia enfatiza a liberdade dos envolvidos, tendo em vista o reconhecimento voluntário de doadores e receptores. Para que isso ocorra, é de suma importância que sejam devidamente informados das eventuais consequências do transplante, para que possam tomar uma decisão de forma consciente. A doação de órgãos poderia ser facilitada se fosse priorizada e garantida uma boa qualidade de comunicação entre os profissionais e a família do doador (SANTOS; MASSAROLLO, 2005; ALMEIDA, 2011).

Por outro lado, não se pode perder de vista a bioética da beneficência, voltada para solidariedade humana, com o intuito de melhoria na problemática que envolve o processo de captação e transplante, cabível seria nesse ampliar meios de campanhas de informação e sensibilização. De certa forma, a ignorância ou a errada compreensão dos aspectos legais que envolvem a ética dos profissionais envolvidos no processo diminui o número de doações (SELLI, 1998; RODRIGUES; SATO, 2002).
O princípio da justiça releva quantas pessoas poderiam eventualmente deleitar-se das atuais tecnologias cientificas com a distribuição equitativa de órgãos disponíveis para o procedimento. Flor (2004) discute que os avanços precisam traduzir-se em direitos materializados e efetivos.

No intuito de direcionar a discussão para o processo de doação de órgãos, criamos casos hipotéticos para realizamos discussões acerca das questões éticas e bioéticas envolvidas. Em seguida, focamos no doador cadáver e discutimos como agir diante deste paciente e seus familiares, balizando-se nos princípios da bioética por entendermos importante a possibilidade do familiar não aceitar a doação de órgãos, pautados na crença de que o familiar está vivo. Foram questões muito presentes na discussão: Como agir diante tal situação? É possível desligar os aparelhos ou suspender o tratamento medicamentoso? Como isso seria interpretado pela família: eutanásia? Caberia processo por parte dos familiares? Tivemos que retomar conceitos sobre ética, bioética, morte e vida para que os participantes pudessem refletir e desvelar criticamente a temática em foco.

Existem ainda muitas dúvidas no que se diz respeito ao que é realmente considerado morte (SANTOS; BUENO, 2010), o que se agrava quando se fala sobre a morte encefálica. A falta de comunicação adequada gera lacunas para mitos e desconfiança sobre a ética dos profissionais, já que o paciente apresenta batimentos cardíacos, movimentos respiratórios e temperatura corpórea. Essa falta de confiança faz com que familiares, e até mesmo aqueles que têm intenções futuras de ser um possível doador de órgãos, mudem de ideia (RODRIGUES; SATO, 2002; SANTOS; MASSAROLLO, 2005). Incluímos a preocupação com a possível comercialização desses órgãos (RODRIGUES; SATO, 2002).

Para finalizar, discutimos a questão da abordagem familiar frente a possibilidade de doação de órgãos. Enfatizamos a importância da ambiência e da escolha do membro da família que deve ser abordado considerando as informações que possuem sobre o estado da pessoa, seu entendimento sobre essa condição, a interpretação que fazem dos resultados dos exames e a confiança nas condutas da equipe de saúde. Procuramos enfocar a discussão sobre evitar a obstinação terapêutica, ou seja, possibilitar aos alunos, futuros profissionais da saúde que poderão atuar nesta área, a compreensão de suas limitações frente ao quadro do paciente/potencial doador.

\section{Desvelamento crítico}

Os círculos de cultura possibilitaram o desvelamento crítico dos fundamentos éticos no processo de doação de órgãos, de forma que os participantes relatavam, durante as discussões, as novas percepções construídas pelo diálogo autêntico entre educandos e educador, configurando o que Paulo Freire denominou de consciência crítica (FREIRE, 2011). O desvelamento crítico foi a todo momento evidenciado, especialmente porque percebemos a superação da visão ingênua que possuíam ao responder ao questionário aplicado no início da pesquisa.

Ao final, os relatórios elaborados pelos alunos fez-nos compreender que, de fato, essa abordagem didática, integrando teoria e crítica pelo diálogo, foi responsável por uma nova apreensão da realidade que envolve o processo de 
doação de órgãos. Pelos relatórios expressarem a sua opinião sobre a atividade desenvolvida e relataram que a ação educativa permitiu alavancar seus saberes no entorno da temática e que reflexões dessa natureza são imprescindíveis para a formação ética do enfermeiro, indissociáveis do saber técnico-científico.

\section{Conclusão}

A doação de órgãos foi abordada de forma mais pontual nas questões bioéticas que permeiam esse processo, a partir do contexto dos envolvidos, colaborando para a formação ética, crítica e contextualizada do futuro enfermeiro. Assim, tornou-se de extrema importância resgatar nas falas dos alunos participantes de nosso estudo os pontos que iam ao encontro dos princípios da bioética e do cuidado, no intuito de evidenciar e resgatar os valores humanos inerentes à vida. A realização de ação educativa, não perdendo o olhar bioético no decorrer das discussões que foram elencadas segundo os pressupostos freirianos, estimulou a construção de novos saberes, superando a visão mágica que os participantes tinham sobre o tema.

Evidenciamos que os pontos que dificultam o processo de doação de órgãos possivelmente poderão ser discutidos durante a formação do graduando e com investimentos na educação permanente. A equipe de saúde necessita de preparo para lidar com as questões que envolvem esse processo, de forma humanista e humanitária, solidária, ética e cidadã, pois atuar frente à morte, a doação e o transplante representam algo difícil tanto para a família quanto para o profissional envolvido, demandando ação educativa nessa temática.

\section{Referências}

ALMEIDA, E. C. Doação de Órgãos e visão da família sobre atuação dos profissionais neste processo: revisão sistemática da literatura brasileira. 2011. 97 f. Dissertação (Mestrado em Ciências) - Escola de Enfermagem de Ribeirão Preto, Universidade de São Paulo, Ribeirão Preto, 2011.

CINQUE, V. M.; BIANCHI, E. R. F. Estressores vivenciados pelos familiares no processo de doação de órgãos e tecidos para transplantes. Rev Esc Enf. São Paulo, v. 44, n. 4, p. 996-1002, 2010.

DINIZ, D.; GUILHEM, D. O que é bioética. São Paulo: Brasiliense, 2002.

FERNANDEZ, J. G. 10 Palavras-chave em bioética. São Paulo: Paulinas, 2000. p. 282-283.

FREIRE, P. Pedagogia do oprimido. 50 ed. Rio de Janeiro: Paz e Terra, 2011.

FLOR, P. E. A. Transplantes: uma leitura constitucional. In: SPAREMBERGER, R. F. L.; AUGUSTIM, S. Direito ambiental e bioética. Caxias do Sul: EDUCS, 2004. p. 157-169.

LIMA, A. A. F. Doação de órgãos para transplante: conflitos éticos na percepção do profissional. Rev. O Mundo da Saúde, São Paulo, v. 36, n.1, p. 27-33, 2012.

Vulnerabilidade dos profissionais de saúde no processo de doação de órgãos para transplante. In: BARCHIFONTAINE, C. P.; ZOBOLI, E. L. C. P. (Org.). Bioética, vulnerabilidade e saúde. Aparecida: Idéias \& Letras, 2007. p. 347-355.

MORAIS, M. R.; MORAIS, T. R. Doação de órgãos: é preciso educar para avançar. Saúde em Debate, Rio de Janeiro, v. 36, p. 633-639, dez. 2012.

OSELKA, G. Bioética clinica: reflexões e discussões sobre casos selecionados. São Paulo: Centro de Bioética, 2008. p. 266.

REICH, W. Análise do caráter. São Paulo: M. Fontes, 1995. p. 491.

RODRIGUES, A. M.; SATO, D. H. Conhecimento e atitude da população do hospital de São Paulo sobre doação de córneas. Arq Bras Oftalmol. v. 65, n. 6, p. 637-640. 2002.

ROZA, B. A.; SCHIRMER, J. A bioética como instrumento para a prática na doação de órgãos e tecidos. In: MALAGUTTI, W. (Org.). Bioética e enfermagem controvérsias, desafios e conquistas. Rio de Janeiro: Rubio, 2007.

SANTOS, J. L.; BUENO, S. M.V. Educação para a morte a docentes e discentes de enfermagem: revisão documental da literatura científica. Rev Esc Enf. USP. v. 45, n. 1, p. 272-276, 2011.

SANTOS, M. J.; MASSAROLLO. M. C. K. B. Processo de doação de órgãos: percepção de familiares de doadores cadáveres. Rev Latino-am Enfermagem, v. 13, n. 3, p. 382-387, 2005

SANTOS, M. J.; MASSAROLLO. M. C. K. B.; MORAES, E. L. Entrevista familiar no processo de doação de órgãos e tecidos para transplante. Acta Paul Enferm, v. 25, n. 5, p. 788-794, 2012.

SELLI, L. Bioética na enfermagem. São Leopoldo: Unisinos, 1998, p. 141.

VIEIRA, T. R. Bioética temas atuais e seus aspectos jurídicos. Brasília: Consulex, 2006. 